\title{
Breast cancer patients with bone metastases are characterised by increased levels of nonisomerised type I collagen fragments
}

\author{
Paul Andreas Compare Cloos ${ }^{1}$, Stephan Christgau1 ${ }^{1}$, Nina Lyubimova ${ }^{2}$, Jean-Jacques Body ${ }^{3}$, \\ Per Qvist ${ }^{1}$ and Claus Christiansen ${ }^{4}$
}

\author{
${ }^{1}$ Nordic Bioscience, Herlev, Denmark \\ ${ }^{2}$ NN Blokhin Cancer Research Centre of RAMS, Moscow, Russia \\ 3Université Libre de Bruxelles, Bruxelles, Belgium \\ ${ }^{4}$ Center for Clinical and Basic Research, Ballerup, Denmark \\ Correspondence: Paul Andreas Compare Cloos (e-mail: PC@nordicbioscience.com)
}

Received: 12 Dec 2002 Revisions requested: 17 Feb 2003 Revisions received: 4 Apr 2003 Accepted: 16 Apr 2003 Published: 19 May 2003

Breast Cancer Res 2003, 5:R103-R109 (DOI 10.1186/bcr607)

(C) 2003 Cloos et al., licensee BioMed Central Ltd (Print ISSN 1465-5411; Online ISSN 1465-542X). This is an Open Access article: verbatim copying and redistribution of this article are permitted in all media for any purpose, provided this notice is preserved along with the article's original URL.

\begin{abstract}
Background Fragments of collagen type I containing the epitope AHDGGR (CTX) are generated during bone resorption. The aspartyl-glycine (DG) site within CTX is synthesised in the L-aspartyl peptide $(\alpha L)$ form, but converts to the age-modified forms L-isoaspartyl peptide $(\beta \mathrm{L})$ and $D$-aspartyl peptide $(\alpha D)$ over time. The purpose of the present study was to test the ability of the various CTX forms to identify breast cancer patients with bone metastases and to investigate whether such patients had an altered CTX excretion pattern.
\end{abstract}

Methods In this cross-sectional study we compared CTX excretion in healthy premenopausal and postmenopausal women with CTX levels in patients with breast cancer. The breast cancer cohort comprised eight hypercalcemic patients with bone metastases $(\mathrm{HC}+), 100$ normocalcemic patients with bone metastases (NC+) and 15 normocalcemic patients without bone metastases (NC-).
Results In $\mathrm{HC}+$ patients and $\mathrm{NC}+$ patients, the excretion of $\alpha \mathrm{L}$ CTX was highly increased compared with NC- patients $(P<0.01)$, with $Z$ scores of 3.4 and 2.0 , respectively. The excretion of the age-modified forms ( $\beta \mathrm{L}$ and $\alpha \mathrm{D} C T X)$ was less increased in $\mathrm{HC}+$ patients and in $\mathrm{NC}+$ patients as compared with NC- patients, with $Z$ scores of 2.2 and 1.0, respectively, for $\beta \mathrm{L} C T X$, and of 1.6 and 0.8 , respectively, for $\alpha D$ CTX.

Conclusion Assays for the various isoforms of CTX have different sensitivities to identify patients affected by bone metastases. The $\alpha \mathrm{L}$ CTX isoform reflecting resorption of young bone appeared to provide the best differentiation of patients affected by breast cancer-induced bone metastases. In conclusion, patients affected by metastatic bone disease present an altered excretion pattern of CTX isoforms.

Keywords: bone metastases, bone resorption, bone turnover, biomarkers, isomerisation

\section{Introduction}

A characteristic feature of many cancer types is their ability to metastasise to the skeleton. At the skeletal site of metastatic invasion, the bone metabolism is dysregulated and bone resorption or bone formation, or both, is upregulated. Bone is the most common target site of metastatic invasion in breast cancer [1]. This is of clinical importance as metastatic bone disease is associated with increased morbidity and excess mortality [2]. To initiate successful preventive therapy it is thus imperative that the bone metastases are detected early [3].

The diagnosis of bone metastases is usually performed through bone scintigraphy. However, this technique is invasive and is not sensitive enough for detection of the very early stages of metastasis [4]. Biochemical markers of bone turnover are noninvasive and may potentially provide a more sensitive measure of metastatic skeletal

$\alpha \mathrm{D}=\mathrm{D}$-aspartyl peptide; $\alpha \mathrm{L}=\mathrm{L}$-aspartyl peptide; $\beta \mathrm{D}=\mathrm{D}$-isoaspartyl peptide; $\beta \mathrm{L}=\mathrm{L}$-isoaspartyl peptide; $\mathrm{Cr}=\mathrm{creatinine} ; \mathrm{CTX}=\mathrm{crosslinked}$ C-telopeptide of type I collagen; ELISA = enzyme-linked immunosorbent assay, $\mathrm{HC}+=$ hypercalcemic with bone metastases; NC+ = normocalcemic with bone metastases; $\mathrm{NC}-=$ normocalcemic without radiological signs of bone metastases; $\mathrm{SD}=$ standard deviation. 


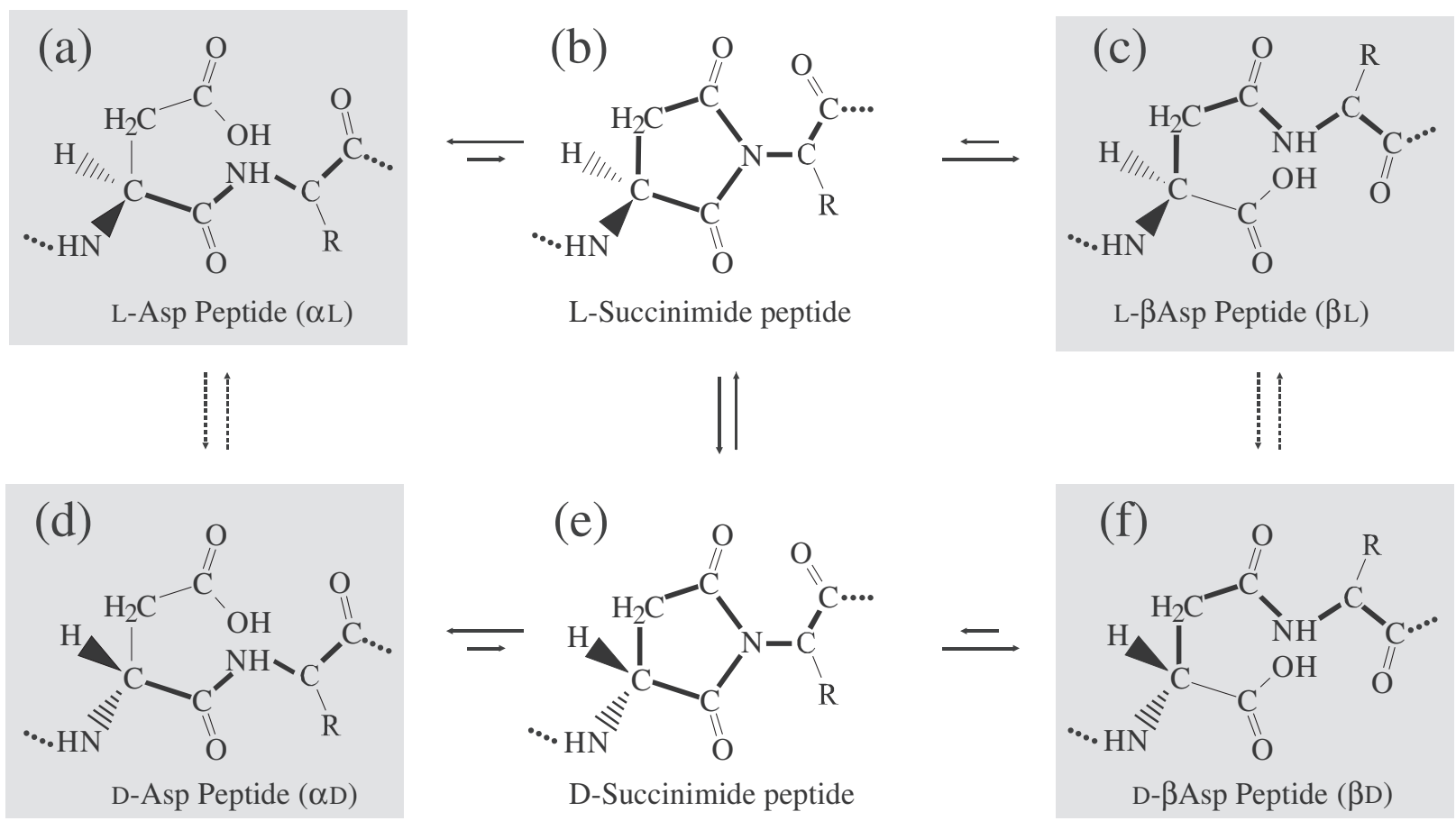

Racemisation and isomerisation of CTX molecules. Reaction of the peptide backbone nitrogen with the aspartyl carboxyl residue can result in the formation of a succinimide ring $(a \rightarrow b)$. This intermediate produces peptides and $\beta$-aspartyl peptides in both the $\mathrm{D}$ and $\mathrm{L}$ configurations. CTX, crosslinked C-telopeptide of type I collagen

invasion, but clinical applications of the bone turnover markers have not been thoroughly evaluated for such a clinical role.

Degradation products derived from the $\alpha 1$ chain of type I collagen (CTX) have been shown to provide a sensitive and specific index of bone resorption $[5,6]$. Preliminary studies have suggested that the CTX marker may be applicable for monitoring skeletal metastases in breast and prostate cancer patients [7,8]. The epitope recognised in the CTX assays comprises the six residues AHDGGR ${ }^{1209-1214}$ of the collagen type I C-terminal telopeptide. The aspartyl-glycine site (DG) within this epitope is susceptible to spontaneous nonenzymatic rearrangement, as outlined in Fig. 1.

CTX fragments derived from newly synthesised collagen are of the native peptide form (L-aspartyl peptide $[\alpha L]$ ), where the aspartic acid residue $\left(D^{1211}\right)$ is on the L-enantiomeric form and is linked to the glycine residue $\left(G^{1212}\right)$ through a peptide bond. Collagen and CTX fragments will rearrange with age to three age-modified forms: the isomerised form (L-isoaspartyl peptide $[\beta\llcorner]$ ), where the bond between the aspartic acid and glycine residue goes through the $\beta$-carboxyl group of the aspartyl residue; the tic acid $\left(D^{1211}\right)$ is on a D-enantiomeric form; and the isomerised and racemised form (D-isoaspartyl peptide $[\beta D]$ ) [9]. The relative accumulation of the three age-modified forms is dependent on the balance between anabolic and catabolic processes in bone, and pathologic situations affecting these processes may thus also affect the relative amounts of the four CTX forms [9-11].

It has been shown that in Paget's disease of bone, a condition characterised by a localised upregulation of bone turnover, there is a significant increase in the ratio between the native peptide form of CTX $(\alpha \mathrm{L})$ and the agemodified forms ( $\beta \mathrm{L}, \mathrm{D}$-isoaspartyl peptide and $\alpha \mathrm{D}$ ) [9]. The use of CTX ratios may thus be indicative of the significant decrease in the residence time and therefore the average age of the resorbed bone collagen type I fragments in Pagetic patients [9]. This indicates that perturbations in the normally well-regulated process of bone turnover can result in changes in the half-life of collagen type I within the bone matrix. This in turn will result in increased amounts of the newly synthesised $\alpha \mathrm{L}$ CTX form in the circulating pool of CTX fragments that is reflected in the elevated ratios [9-11].

Metastatic bone cancer will also affect the balance between the anabolic and catabolic processes at the local 
site of invasion. The measurement of CTX isoforms, and the ratios between the native $\alpha \mathrm{L}$ form and the age-modified forms of CTX, indicative of the age of the resorbed collagen fragments, may thus provide clinical useful information for diagnosing and monitoring bone metastasis occurrence in cancer patients [11]. Such an application of the CTX bone resorption markers may be especially relevant for breast cancer patients, who have a high risk of developing bone metastases.

In the present study, our aim was to assess the clinical utility of the different CTX isoforms, as well as the ratios between the isoforms, to detect breast cancer-induced bone metastases. For this purpose, the urinary CTX isoforms $\alpha \mathrm{L}, \beta \mathrm{L}$, and $\alpha \mathrm{D}$ were quantified by immunoassay in healthy premenopausal and postmenopausal women, and in women affected with breast cancer either with or without bone metastases.

\section{Patients and methods Study population}

Urine samples were collected as the morning void from 24 healthy premenopausal women (age [mean \pm standard deviation (SD)], $33.9 \pm 5.6$ years) and 31 healthy postmenopausal women (age 55.1 \pm 2.7 years). In addition, 123 female patients with histologically confirmed breast cancer participated in the study. Eight patients were hypercalcemic (serum calcium $>2.7 \mathrm{mmol} / /$, albumin corrected), had radiologically confirmed bone metastases and were $54.4 \pm 12.0$ years of age ( $\mathrm{HC}+$ patients). One hundred patients were normocalcemic (serum calcium between 2.0 and $2.7 \mathrm{mmol} / \mathrm{l}$, albumin corrected), had radiologically confirmed bone metastases and were $54.4 \pm 12.0$ years of age (NC+ patients). Finally, 15 breast cancer patients ( $54.5 \pm 12.3$ years of age) were normocalcemic, had early stage (nonmetastatic) cancer but were without radiological signs of bone metastases (NCpatients).

Fifty per cent, $49 \%$ and $53 \%$ of subjects were premenopausal in the $\mathrm{HC}+, \mathrm{NC}+$ and $\mathrm{NC}-$ patient groups, respectively. There was no statistical difference between the proportions of premenopausal women in the three groups.

The presence or absence of bone metastasis in patients was determined by bone scintigraphy, and was confirmed by conventional radiography (X-ray) as necessary. All study subjects had serum creatinine $<265 \mu \mathrm{mol} / \mathrm{l}$, and received endocrine therapy and/or an anthracycline-containing regimen. Some patients had additional radiotherapy in the event of clinical symptoms (e.g. bone pain, instability). The use of antineoplastic hormonal treatment $(20 \mathrm{mg}$ tamoxifen, $500 \mathrm{mg}$ aminoglutethimide, $500 \mathrm{mg}$ provera) was allowed, but none of the patients were receiving bisphosphonate therapy prior to entry into the study. Informed, written consent was obtained from all par- ticipants according to the Helsinki Declaration of 1975 , as revised in 1983. The study was approved by the local human investigations committee.

\section{Measurement of CTX isoforms by immunoassay analysis}

The $\alpha$-CrossLaps radioimmunoassay [12] and the CrossLaps ELISA [13] are commercially available immunoassays specific for the native $(\alpha \mathrm{L})$ and isomerised $(\beta \mathrm{L})$ forms of CTX, respectively. The assays were performed as recommended by the manufacturer (Nordic Bioscience Diagnostics, Herlev, Denmark).

The $\alpha D$-CTX ELISA is a competitive assay based on polyclonal rabbit antibodies for the $\alpha D$ form of CTX. The assay has been described elsewhere [9]. All determinations of urinary CTX were corrected for creatinine. The three CTX assays were calibrated using synthetic CTX standards, the concentration of which had been determined by analysis of the total amino acid composition as described previously [14].

\section{Statistical analysis}

Biochemical data were compared between groups using the Mann-Whitney $U$ test (two-tailed). For all tests $P \leq 0.05$ was considered significant. The discriminatory power of the different CTX isoforms or CTX ratios in distinguishing groups from controls was assessed using $T$ or $Z$ scores (i.e. number of SDs from the mean of the controls).

\section{Results}

The urinary excretion of all CTX forms was significantly higher $(P<0.001)$ in the postmenopausal group when compared with the premenopausal women, with approximately 2.5-fold higher levels (Table 1 ). When ratios between the newly synthesised $\alpha \mathrm{L}$ form and the age-modified forms $(\beta\llcorner$ and $\alpha D)$ were calculated, only minor and nonsignificant differences were observed between premenopausal and postmenopausal women. In patients with breast cancer-induced bone metastases, the urinary excretion of the newly synthesised $\alpha \mathrm{L}$ CTX form was more increased than the age-modified forms $\beta \mathrm{L}$ and $\alpha \mathrm{D}$. Compared with NC- patients, the $\mathrm{HC}+$ patients had a 3.4-fold elevation in $\alpha \mathrm{L} C T X$ and the NC+ patients had 2.2-fold higher levels of $\alpha \mathrm{L}$ CTX $(P<0.01)$. The levels of $\alpha \mathrm{D}$ CTX, representing the oldest form of CTX, were increased 2.4-fold and 1.7-fold in $\mathrm{HC}+$ patients and in $\mathrm{NC}+$ patients compared with NC- patients (Fig. 2). The ratio between the nonisomerised $\alpha L$ form and the racemised $\alpha D$ form of CTX was consequently increased in NC+ patients and in $\mathrm{HC}+$ patients 2.2 and 1.5 times, respectively, when compared with NC- patients, and increased 2.4 and 1.6 times, respectively, compared with postmenopausal patients. However, these increases in the $\alpha \mathrm{L} / \alpha \mathrm{D}$ ratio did not reach statistical significance as evaluated by nonparametric statistics. 
Table 1

CTX levels in different groups

\begin{tabular}{|c|c|c|c|c|}
\hline \multirow[b]{2}{*}{ Subjects } & & \multicolumn{3}{|c|}{ CTX isoform } \\
\hline & & $\alpha\llcorner C T X / C r$ & $\beta\llcorner\mathrm{CTX} / \mathrm{Cr}$ & $\alpha \mathrm{D} C T X / \mathrm{Cr}$ \\
\hline Premenopausal patients & Mean \pm standard deviation & $3.65 \pm 1.41$ & $5.74 \pm 3.46$ & $1.75 \pm 0.94$ \\
\hline Postmenopausal patients & $\begin{array}{l}\text { Mean } \pm \text { standard deviation } \\
P, \cup \text { testa } \\
T \text { score }^{\mathrm{a}}\end{array}$ & $\begin{array}{c}8.39 \pm 3.01 \\
<0.001 \\
3.4\end{array}$ & $\begin{array}{c}13.6 \pm 3.9 \\
<0.001 \\
2.3\end{array}$ & $\begin{array}{c}3.62 \pm 1.08 \\
<0.001 \\
2.0\end{array}$ \\
\hline $\begin{array}{l}\text { Normocalcemic breast cancer } \\
\text { patients without bone metastases }\end{array}$ & $\begin{array}{l}\text { Mean } \pm \text { standard deviation } \\
P, \cup \text { test }^{\mathrm{b}} \\
Z \text { score }^{\mathrm{b}}\end{array}$ & $\begin{array}{c}10.2 \pm 6.23 \\
\text { Not significant } \\
0.60\end{array}$ & $\begin{array}{c}12.7 \pm 11.3 \\
\text { Not significant } \\
-0.23\end{array}$ & $\begin{array}{c}4.73 \pm 4.14 \\
\text { Not significant } \\
1.03\end{array}$ \\
\hline $\begin{array}{l}\text { Normocalcemic breast cancer } \\
\text { patients with bone metastases }\end{array}$ & $\begin{array}{l}\text { Mean } \pm \text { standard deviation } \\
P, \mathrm{U} \text { test }^{\mathrm{c}} \\
Z \text { score }^{\mathrm{c}}\end{array}$ & $\begin{array}{l}22.6 \pm 24.8 \\
<0.01 \\
2.00\end{array}$ & $\begin{array}{l}24.6 \pm 18.4 \\
<0.01 \\
1.01\end{array}$ & $\begin{array}{l}7.95 \pm 9.11 \\
<0.05 \\
0.76\end{array}$ \\
\hline $\begin{array}{l}\text { Hypercalcemic breast cancer } \\
\text { patients with bone metastases }\end{array}$ & $\begin{array}{l}\text { Mean } \pm \text { standard deviation } \\
P, \mathrm{U} \text { test }^{\mathrm{c}} \\
Z \text { score }^{\mathrm{c}}\end{array}$ & $\begin{array}{l}35.1 \pm 27.8 \\
\quad<0.01 \\
3.41\end{array}$ & $\begin{array}{l}38.0 \pm 23.7 \\
<0.01 \\
2.24\end{array}$ & $\begin{array}{l}11.3 \pm 10.2 \\
<0.05 \\
1.59\end{array}$ \\
\hline
\end{tabular}

Concentrations are provided as $\mu \mathrm{mol} / \mathrm{mol} . \alpha \mathrm{D}=\mathrm{D}$-aspartyl peptide; $\alpha \mathrm{L}=\mathrm{L}$-aspartyl peptide; $\beta \mathrm{L}=\mathrm{L}$-isoaspartyl peptide; Cr, creatinine; CTX, crosslinked C-telopeptide of type I collagen. aVersus premenopausal patients. ${ }^{b}$ Versus postmenopausal patients. ${ }^{c}$ Versus normocalcemic breast cancer patients without bone metastases.

The ability of the various CTX forms and CTX ratios to discriminate between patients with bone metastases $(\mathrm{HC}+$ patients and $\mathrm{NC}+$ patients) and patients without bone metastases (NC- patients) was assessed by Z-score analysis using the mean and SD of the NC- patient group (Fig. 3). This analysis revealed that the $\alpha$ L CTX levels provided the best differentiation between patients with metastases ( $\mathrm{HC}+$ patients and $\mathrm{NC}+$ patients) and nonmetastasised controls (Fig. 3).

Although the $\alpha L / \alpha D$ ratio displayed the highest mean $Z$ score, the urinary excretion of the $\alpha L$ CTX form still appeared to have the best ability to identify patients affected by bone metastases. Thus, 58\% of patients affected by bone metastases had significantly increased $\alpha L$ CTX values ( $Z$ score $>2$ ) compared with breast cancer patients without bone metastases (NC-patients) (Fig. 3). In comparison, $30 \%$ of patients affected by bone metastases had $\alpha \mathrm{L} / \alpha \mathrm{D}$ ratios more than 2 SDs above the normal postmenopausal mean (Fig.3). In addition, whereas only $27 \%$ of patients affected by bone metastases had $\alpha \mathrm{L}$ CTX values below the mean of nonmetastasised controls (NCpatients), $52 \%$ of these patients had $\alpha L / \alpha D$ ratios below this value (Fig. 3).

\section{Discussion}

In the present study, the clinical utility of assays for the various CTX forms in the assessment of breast cancerinduced bone metastases was investigated.

The urinary concentration of all four CTX isoforms was initially measured in reference populations of healthy pre- the three CTX isoforms $\alpha \mathrm{L}, \beta \mathrm{L}$ and $\alpha \mathrm{D}$ were 2.3-fold to 2.7-fold higher in the postmenopausal women (Table 1), in accordance with previous reports of CTX levels during the menopause [15]. As all forms were elevated to the same magnitude in postmenopause, the ratios between the newly synthesised $\alpha L$ CTX form and the older $\beta L$ and $\alpha D$ CTX forms was not significantly different in premenopausal and postmenopausal women. This indicates that the average 'age' and thus the half-life of bone collagen is similar in premenopausal and postmenopausal women. In a study comprising larger cohorts, a small but significant elevation in the $\alpha \mathrm{L} / \beta \mathrm{L}$ ratio was found, indicating that the net half-life of collagen is slightly decreased after the menopause [16].

In breast cancer patients affected by bone metastases ( $\mathrm{HC}+$ patients and $\mathrm{NC}+$ patients), the levels of all CTX isoforms were elevated compared with healthy postmenopausal controls or with NC- patients. The $\alpha \mathrm{L}$ form representing the degradation of newly formed bone was, however, more elevated than the corresponding agerelated isoforms (Table 1). $\mathrm{HC}+$ patients had a 3.4-fold elevation in $\alpha L C T X$ levels, and a 2.2-fold elevation was seen in $\mathrm{NC}+$ patients, indicating that the more severe condition in the $\mathrm{HC}+$ patients is reflected by the $\alpha \mathrm{L}$ CTX marker. The least prominent elevation was seen for the $\alpha \mathrm{D}$ CTX levels.

The $\alpha L / \alpha D$ CTX ratio reflecting the relative contents of age-modified CTX forms was highly increased in $\mathrm{HC}+$ patients (1.5-fold and 1.6-fold compared with NCpatients and with postmenopausal patients, respectively) and in NC+ patients (2.2-fold and 2.4-fold compared with NC- patients and with postmenopausal patients). This 
Figure 2
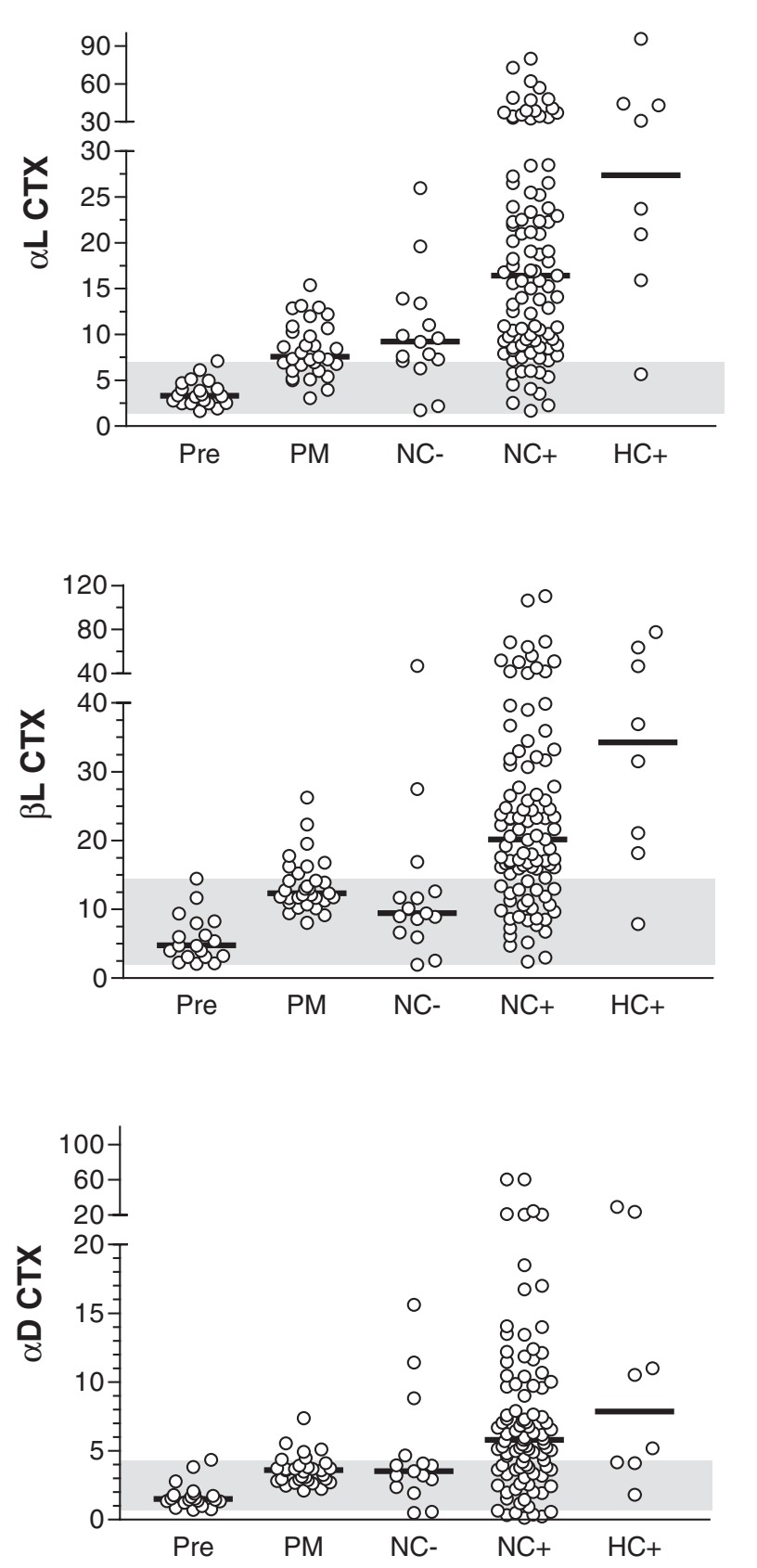

Excretion of CTX isoforms in healthy premenopausal (Pre) and postmenopausal controls (PM), and in breast cancer patients with hypercalcemia and bone metastases $(\mathrm{HC}+)$, in normocalcemic patients with bone metastases ( $\mathrm{NC}+$ ) and in normocalcemic patients without bone metastases (NC-). Shaded areas indicate mean \pm 2 standard deviations for healthy premenopausal women. Bars indicate medians. $\alpha \mathrm{D}=\mathrm{D}$-aspartyl peptide; $\alpha \mathrm{L}=\mathrm{L}$-aspartyl peptide; $\beta \mathrm{L}=\mathrm{L}-$ isoaspartyl peptide; CTX, crosslinked C-telopeptide of type I collagen.

suggests that the age and thus the half-life of collagen type I fragments generated by resorption is decreased in the group of patients with bone metastases. Such a decrease in collagen type I half-life is likely to reflect a sig-
Figure 3

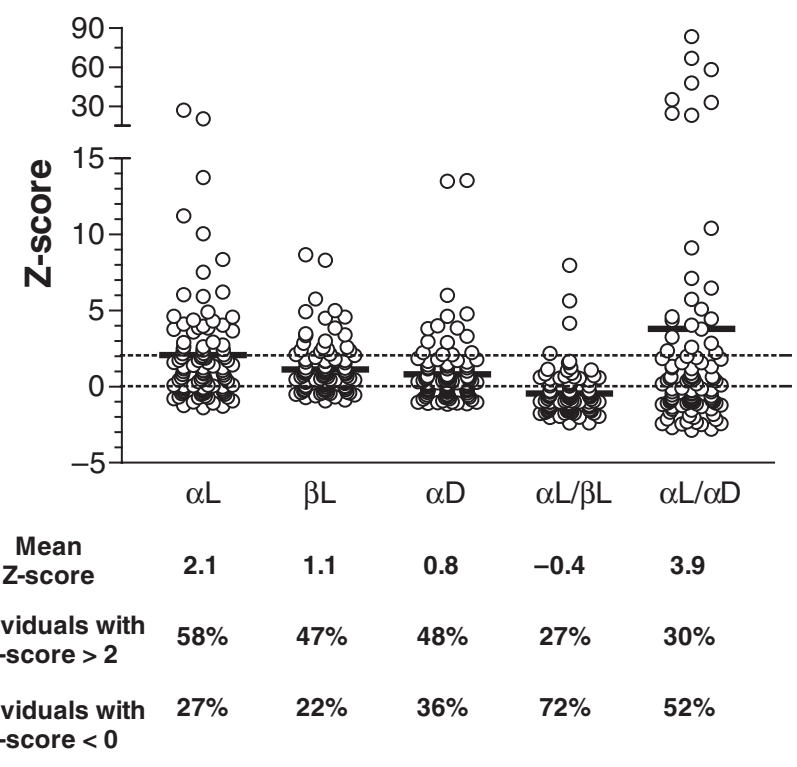

The discriminatory power of CTX markers and CTX ratios to identify breast cancer-induced bone metastases. Open circles, individual $Z$ scores for hypercalcemic patients with bone metastases $(\mathrm{HC}+)$ and normocalcemic patients with bone metastases $(\mathrm{NC}+)$. $Z$ scores are calculated using the mean and standard deviation of normocalcemic breast cancer controls without bone metastases. $\alpha D=D$-aspartyl peptide; $\alpha \mathrm{L}=\mathrm{L}$-aspartyl peptide; $\beta \mathrm{L}=\mathrm{L}$-isoaspartyl peptide; CTX, crosslinked C-telopeptide of type I collagen.

nificant imbalance between anabolic and catabolic processes at the focal area of metastatic invasion [11].

A similar imbalance is seen in some 'high-turnover' conditions, including Paget's disease [9,17]. In situations of high bone turnover, the 'aged' bone matrix will rapidly be replaced with a new matrix, leading to a lower residence time (i.e. half-life) of bone collagen. More 'young bone' (as reflected by the $\alpha L$ CTX levels) will be resorbed and the relative content of age-modified forms will decrease. The relative content of $\alpha L$ CTX is reflected in the CTX ratios, providing an index of bone collagen half-life [9]. Among the CTX ratios, the $\alpha L / \alpha D$ ratio showed the most pronounced elevation in the metastatic breast cancer patients. This is in good agreement with kinetic studies of the isomerisation and racemisation reactions, where it was shown that the $\alpha D$ CTX form accumulates with the lowest kinetic rate [9]. The $\alpha\llcorner/ \alpha D$ CTX ratio will thus be most affected by changes of bone turnover processes influencing the half-life of collagen type I in the bone matrix. The age-related $\beta \mathrm{L}$ form accumulates with a slightly higher kinetic rate than $\alpha D$ CTX [9]. This is also apparent from the less pronounced increase in the $\alpha \mathrm{L} / \beta \mathrm{L}$ ratio compared with the $\alpha L / \alpha D$ ratio in metastatic patients.

When individual $Z$ scores of CTX were calculated for the metastatic breast cancer patients compared with the non- 
metastatic patients, the $\alpha \mathrm{L}$ CTX form and the $\alpha \mathrm{L} / \alpha \mathrm{D}$ CTX ratio showed the highest average increases in accordance with the ability of the $\alpha \mathrm{L}$ CTX levels and the $\alpha \mathrm{L} / \alpha \mathrm{D}$ CTX ratio to most sensitively reflect alterations in the balance between the anabolic and catabolic processes of bone turnover (Fig. 3). $\alpha$ L CTX levels alone showed a significant elevation in $58 \%$ of the metastatic patients, which probably reflects an increased bone turnover (increased resorption + increased formation) as an important feature in the majority of bone metastatic patients. For the $\beta \mathrm{L}$ and $\alpha \mathrm{D}$ CTX markers, respectively, 47 and $48 \%$ of the metastatic patients had levels 2 SDs above the reference level of nonmetastasised controls (Fig. 3).

The present data suggest that the levels of $\alpha L$ CTX are most affected by the altered bone turnover associated with metastatic invasion of bone. The fact that only $58 \%$ of patients affected by bone metastases (as determined by radiology) have increased levels of $\alpha \mathrm{L}$ CTX indicates a rather low sensitivity of bone resorption markers to identify bone metastases. Several issues are relevant for the interpretation of this result. It may be speculated that different stages of the bone metastases process show varying release of $\alpha \mathrm{L}$ CTX. Furthermore, the difference may be related to differences between sclerotic and lytic bone metastases. In this context it is noteworthy that some patients who have not been classified as having bone metastases by radiological methods have $\alpha \mathrm{L}$ CTX values increased more than 2 SDs above the normal mean.

High levels of $\alpha L$ CTX are indicative of an increased bone resorption. In the clinical management of individual patients affected with breast cancer, an increased $\alpha \mathrm{L}$ CTX value may indicate the presence of bone metastases and will call for a closer clinical evaluation of the patient and/or initiation of preventive therapy (e.g. bisphosphonates).

The study has some limitations. Most patients received antineoplastic hormonal treatment $(20 \mathrm{mg}$ tamoxifen, $500 \mathrm{mg}$ aminoglutethimide, $500 \mathrm{mg}$ provera). This treatment may potentially reduce bone resorption, thereby influencing CTX levels. It may thus be a confounding factor in the analysis. In spite of this treatment, however, patients affected by bone metastases had highly increased marker values compared with healthy controls. This indicates that the increase in markers is related to metastatic invasion of bone and is reflected in systemic marker levels regardless of the potential antiresorptive effects of antineoplastic therapy.

\section{Conclusion}

Assays for the various age-related isoforms of CTX have different sensitivities to identify patients affected by bone metastases. The $\alpha L C T X$ isoform reflecting resorption of young bone appeared to provide the best differentiation of tases. Further studies are needed to investigate the utility of this marker in the early detection of bone metastases and for assessing treatment efficacy. Such studies should correlate marker values with metastasis type (lytic, sclerotic, mixed) and with the volume of bone affected by metastases.

\section{Competing interests}

Paul Cloos, Stephan Christgau and Per Qvist are employees of Nordic Bioscience A/S, which commercializes the CTX immunoassays. Per Qvist and Claus Christiansen own shares in Nordic Bioscience A/S. Jean-Jacques Body and Nina Lyubimova have no competing interests.

\section{References}

1. Solomayer EF, Diel IJ, Meyberg GC, Gollan C, Bastert G: Metastatic breast cancer: clinical course, prognosis and therapy related to the first site of metastasis. Breast Cancer Res Treat 2000, 59:271-278.

2. Body JJ, Bartl R, Burckhardt P, Delmas PD, Diel IJ, Fleisch H, Kanis JA, Kyle RA, Mundy GR, Paterson AH, Rubens RD: Current use of bisphosphonates in oncology. International Bone and Cancer Study Group. J Clin Oncol 1998, 16:3890-3899.

3. Niccolini A, Carpi A: Postoperative follow-up of breast cancer patients: overview and progress in the use of tumor markers. Tumor Biol 2000, 21:235-248.

4. Dillman RO, Chico S: Radiologic test after new diagnosis of breast cancer. Eff Clin Pract 2000, 3:1-6.

5. Christgau S, Rosenquist $\mathrm{C}$, Alexandersen $\mathrm{P}$, Bjarnason NH, Ravn $P$, Fledelius C, Herling C, Qvist $P$, Christiansen C: Clinical evaluation of the Serum CrossLaps One Step ELISA, a new assay measuring the serum concentration of bone-derived degradation products of type I collagen C-telopeptides. Clin Chem 1998, 44:2290-2300.

6. Rosen HN, Moses AC, Garber J, lloputaife ID, Ross DS, Lee SL, Greenspan SL: Serum CTX: a new marker of bone resorption that shows treatment effect more often than other markers because of low coefficient of variability and large changes with bisphosphonate therapy. Calcif Tissue Int 2000, 66:100103.

7. Nguyen-Pamart M, Caty A, Feutrie ML, Fournier C, Gosselin P, Mazeman E: The diagnostic value of urinary crosslaps and serum alkaline phosphatase in patients with prostate cancer. $\mathrm{Br} J$ Urol 1997, 80:452-455.

8. Coleman RE, Purohit OP, Black C, Vinholes JJ, Schlosser K, Huss $\mathrm{H}$, Quinn KJ, Kanis J: Double-blind, randomised, placebo-controlled, dose-finding study of oral ibandronate in patients with metastatic bone disease. Ann Oncol 1999, 10:311-316.

9. Cloos PAC, Fledelius C: Collagen fragments in urine derived from bone resorption are highly racemized and isomerized. A biological clock of protein ageing with clinical potential. Biochem J 2000, 345:473-480.

10. Cloos PAC, Christgau S: Non-enzymatic covalent modifications of proteins: mechanism, physiological consequences and clinical applications. Matrix Biol 2002, 21:39-52.

11. Cloos PAC, Fledelius C, Christgau S, Christiansen C, Engsig M, Delmas P, Body JJ, Garnero P: Investigation of bone disease using isomerized and racemized fragments of type I collagen. Calcif Tissue Int 2002, 72:8-17.

12. Bonde M, Fledelius C, Qvist P, Christiansen C: Coated-tube radioimmunoassay for $\mathrm{C}$-telopeptides of type I collagen to assess bone resorption. Clin Chem 1996, 42:1639-1644.

13. Bonde M, Qvist $P$, Fledelius C, Riis BJ, Christiansen C: Immunoassay for quantifying type I collagen degradation products in urine evaluated. Clin Chem 1994, 40:2022-2025.

14. Barkholdt $\mathrm{V}$, Jensen $\mathrm{AL}$ : Amino acid analysis: determination of cysteine plus half-cysteine in proteins after hydrochloric acid hydrolysis with a disulfide compound as additive. Anal Biochem 1989, 177:318-322.

15. Ravn P, Fledelius C, Rosenquist C, Overgaard K, Christiansen C: High bone turnover is associated with low bone mass in both pre- and postmenopausal women. Bone 1996, 19:291-298. 
16. Reginster JY, Henrotin Y, Christiansen C, Gamwell-Henriksen E, Bruyere O, Collette J, Christgau S: Bone resorption in postmenopausal women with normal and low BMD assessed with biochemical markers specific for telopeptide derived degradation products of collagen type I. Calcif Tissue Int 2001, 69:130-137.

17. Garnero P, Fledelius C, Gineyts E, Serre CM, Vignot E, Delmas PD: Decreased beta-isomerization of the C-terminal telopeptide of type I collagen alpha 1 chain in Paget's disease of bone. J Bone Miner Res 1997, 12:1407-1415.

\section{Correspondence}

Paul Andreas Compare Cloos, Nordic Bioscience, Osteopark, Herlev Hovedgade 207, DK-2730 Herlev, Denmark. Tel: +45 44547732; fax: +45 44548832; e-mail: PC@nordicbioscience.com 